VeritasE Scientia

Vol. 8. $\mathrm{N}^{\circ} 1$.

Enero - Junio del 2019.

ISSN Edición Online: 2617-0639

https://doi.org/10.47796/ves.v8i1.108

\title{
PROCESO DE PROGRAMACIÓN PRESUPUESTARIA Y SU INFLUENCIA EN EL USO DE LOS FONDOS PÚBLICOS EN LA MUNICIPALIDAD DISTRITAL DE ITE, 2017
}

PROCESS OF BUDGETARY PROGRAMMING AND ITS INFLUENCE ON THE USE OF PUBLIC FUNDS IN THE ITE DISTRITAL MUNICIPALITY, 2017

Ederth Christian Alca Tito ${ }^{1}$

Elizabeth I. Medina Soto

Presentado: 03/04/2019

Aceptado: $21 / 06 / 2019$

Publicado online:17/07/2019

\section{RESUMEN}

El propósito del estudio fue determinar de qué manera el proceso de programación presupuestaria influye en el uso de los fondos públicos en la Municipalidad Distrital de Ite, 2017. El nivel de investigación fue explicativo. La población estuvo constituida por los registros de la programación presupuestaria y el uso de los fondos públicos, del año 2017, siendo un total de 60 registros. No fue necesario obtener muestra. El instrumento utilizado fue la guía de análisis documental. Los resultados determinaron que el proceso de programación presupuestaria influye significativamente en el uso de los fondos públicos en la Municipalidad Distrital de Ite, 2017, debido a que en el presupuesto programado y ejecutado de ingresos y gastos por fuente de financiamiento en soles correspondiente al ejercicio presupuestal 2017 de la Municipalidad Distrital de Ite, en lo que se refiere al presupuesto de ingresos programado se tiene en PIM S/. 26347155 soles y lográndose una recaudación de S/. 27303756 soles, que representa el 103.6\% Ilegando a superar a lo programado; en cuanto a los gastos se programó en PIM S/. 26347155 soles, llegándose a ejecutar S/. 20139382 soles, que representa el $76,4 \%$ del presupuesto programado.

Palabras clave: programación presupuestaria, uso de fondos públicos, PIA, PIM, Presupuesto de ingresos, Presupuesto de egresos.

\begin{abstract}
Otherwise they will be observed by the Institutional Control Body; Therefore, the purpose of the study is to: Determine how the budget programming process influences the use of public funds in the District Municipality of Ite, 2017. The level of investigation is explanatory. The
\end{abstract}

\footnotetext{
${ }^{1}$ Maestro en Contabilidad, Tributación y Auditoría

${ }^{2}$ Magíster Académica de la Facultad de Ciencias Empresariales y jurídicas de la Universidad Nacional Jorge Basadre Grohmann de Tacna.
} 
population was constituted by the registries of the budgetary programming and the use of the public funds, of the year 2017, being a total of 60 registries. It was not necessary to obtain a sample. The instrument used was the document analysis guide. The results determined that the budgetary programming process significantly influences the use of public funds in the District Municipality of Ite, 2017, due to the fact that in the programmed and executed budget of income and expenses by source of financing in soles corresponding to the budget year 2017 of the District Municipality of Ite, in what refers to the programmed income budget is in PIM S /. 26347155 soles and a collection of S /. 27303756 soles, which represents 103.6\%, exceeding the amount programmed; in terms of expenses, it was programmed in PIM S /. 26347155 soles, reaching S /. 20139382 soles, which represents $76.4 \%$ of the programmed budget.

Key words: budget programming, use of public funds, PIA, PIM, income budget, expenditure budget.

\section{INTRODUCCIÓN}

En Perú, una de las fases más fundamentales en el proceso presupuestario es la programación presupuestaria, que es cuando las instituciones públicas programan su propuesta de presupuesto institucional y el Ministerio de Economía y Finanzas, elabora el anteproyecto de Presupuesto del Sector Público teniendo en cuenta las referidas propuestas. Para ello, se debe revisar la escala de prioridades de la entidad, se determina la demanda Global de Gasto, donde se incluye la cuantificación de las metas, programas y proyectos, con el fin de conseguir los objetivos institucionales, así como también, es primordial que en esta etapa se determine el financiamiento de la demanda global de gasto, en función a la asignación presupuestaria total. A nivel nacional, cada vez se evidencia que no hay un buen uso de los fondos públicos del Estado, prueba de ello, es que actualmente, algunas autoridades se encuentran con procesos penales por malversación de fondos. Como se tiene conocimiento, en el proceso de programación se estiman los gastos a ser ejecutados en el año fiscal siguiente, en función a los servicios que presta y para el logro de resultados. Al respecto, Apaza (2010) indica que la mayoría de las instituciones públicas tienen dificultades en la programación presupuestari. En la Municipalidad Distrital de Ite, se observa de igual forma, en algunas ocasiones, que no se desarrolla una correcta programación presupuestaria, como por ejemplo no se revisa en su totalidad la Escala de Prioridades de la entidad, no se estima óptimamente los fondos públicos disponibles para el financiamiento del presupuesto anual.

Gancino (2010) concluye que la planificación presupuestaria que se desarrolla sin el personal preparado, solo que se basan en la experiencia, no contribuye a que desarrollen sus actividades en forma eficaz. Matamoros (2013) refiere que la planificación contribuye a que el ente municipal tenga mejores resultados; especialmente en temas presupuestarios, planificación del mantenimiento y de la ejecución de los proyectos y en el cumplimiento de los objetivos y planes trazados, de tal forma que se tome las acciones correctivas en el momento adecuado. Montejo y Arones (2014) en su estudio encontró inconsistencias en relación a la programación y las modificaciones presupuestarias. Mamani (2016) refiere que muchas autoridades municipales en la actualidad en Perú no desarrollan un buen manejo de los fondos públicos que coadyuven a que se concretice la eficacia y eficiencia del uso de los fondos públicos, cumpliendo las normas generales de tesorería, y de esta forma no sea sujeto a observaciones por los Órganos de Control Institucional. Lozano (2016) afirma que la programación presupuestaria es un proceso crucial para el desarrollo de una buena ejecución presupuestal, por lo que es primordial que las unidades orgánicas desarrollen adecuados requerimientos, que se considere en el plan operativo en forma 
correcta, para que no haya modificaciones. Coaquira (2017) determina que todas las unidades orgánicas requieren desarrollar en forma eficiente sus actividades, sin embargo, no le asignan en forma correcta el presupuesto, debido a que no hay una correcta programación del presupuesto, pues a veces se asigna por criterios políticos. De la Cruz (2018) ha evidenciado un mal nivel de ejecución presupuestaria en gasto de inversión a través de los años, con nivel de efectividad que no supera el 50 \%; debido a que no han hecho una adecuada programación, por lo que posteriormente ha habido varias modificaciones presupuestales.

El Sistema Nacional de Presupuesto, es uno de los sistemas administrativos integrantes de la Administración Financiera del Sector Público. Es la agrupación de órganos, normas y procedimientos que conducen el proceso presupuestario de todas las entidades y organismos del Sector Público en sus fases de programación, formulación, aprobación, ejecución y evaluación (Ley № 28411, 2011). Establece los límites de gastos durante el año fiscal, por cada una de las Entidades del Sector Público y los ingresos que los financian, acorde con la disponibilidad de los Fondos Públicos, a fin de mantener el equilibrio fiscal (Andía, 2011). El plan estratégico es una de las herramientas esenciales en el manejo presupuestal. Así mismo Lumpkin y Dess (2003), citado por Sainz de Vicuña, (2015), menciona que el plan estratégico como conjunto de actividades, análisis y toma de decisiones que toda institución tiene que realizar con la finalidad de creer y conservar las ventajas competitivas en el tiempo. Vicuña, (2015), refiere que el plan estratégico es un proyecto en donde se realiza el diagnóstico situacional de la organización, incluye las estrategias, actividades a realizar y ver los recursos necesarios para ejecutar dichas actividades y cumplir con los objetivos planteados. Para el CEPLAN (2016, p. 69), se define las acciones que se realizarán, pero en base al presupuesto. De acuerdo a Ruly (2012), el plan operativo institucional es una herramienta de gestión que se utiliza a corto plazo, en donde se precisa las acciones a realizar en plazo de un año. Según Mamani (2016) el presupuesto inicial de toda institución que es aprobada por su respectivo Titular establecidos en la Ley Anual de Presupuesto del Sector Público para el año fiscal respectivo.

Existen ciertas fuentes de financiamiento según Ley General del Sistema Nacional de Presupuesto (Ley $N^{\circ}$ 28411, 2014), recursos ordinarios, recursos directamente recaudados, donaciones, transferencias y recursos determinados. Canon y sobrecanon, son los ingresos que una entidad debe recibir basado en las políticas y normas específicas, por la utilización de bienes naturales que se saca de un determinado lugar. También hacer referencia que incluye los recursos correspondientes a los fideicomisos regionales, y todos los recursos que correspondan de acuerdo a ley (Villegas, 2016).

Los sistemas administrativos, tienen como propósito que las instituciones del Estado consiguen adecuados grados de eficiencia y eficacia. (Andía, 2011). De acuerdo a Ayala y Pineda (2016), los Gobiernos Locales Según la Ley Orgánica de Municipalidades N²7972 los gobiernos locales son entidades básicas de la organización territorial del Estado y canales inmediatos de participación vecinal en los asuntos públicos, que institucionalizan y gestionan con autonomía los intereses propios de la correspondiente colectividad siendo elementos esenciales del gobierno local, el territorio, la población y la organización. Las Municipalidades provinciales y distritales son los órganos de gobierno promotores del desarrollo local con personería jurídica de derecho público y plena capacidad para el cumplimiento de sus fines. Las municipalidades como órganos del gobierno local, tienen asignados entre otras funciones la organización, reglamentación y en su caso la administración de los servicios públicos de su responsabilidad como: la planificación del desarrollo urbano y rural y ejecución de obras de infraestructura local para satisfacer las crecientes necesidades de la población de nuestro país. El desarrollo de estas funciones requiere de financiamiento, es por ello conveniente revisar la normatividad sobre rentas municipales vigentes, para medir el grado de capacidad de gastos y posibilidades de buscar otras fuentes de financiamiento, como es el endeudamiento o las donaciones. (Nueva Ley Orgánica de Municipalidades "Ley N²7972", 2010). 


\section{OBJETIVO}

Establecer cómo el presupuesto programado y ejecutado de ingresos y gastos influye en el uso de los fondos públicos: recursos directamente recaudados, recursos por operaciones, donaciones y transferencias, fondo de compensación municipal, impuesto municipal y Canon y sobrecanon en la Municipalidad Distrital de Ite, 2017.

\section{METODOLOGÍA}

El presente trabajo de investigación es considerado una investigación de tipo básica, diseño no experimental, transversal. El nivel de investigación es explicativo. Ámbito de la investigación es la Municipalidad Distrital de Ite. La unidad de análisis son los registros de programación presupuestaria y manejo de fondos de la Municipalidad Distrital de Ite. Revisión de fuentes documentarias referente a la programación presupuestaria y el uso de fondos públicos (ejecución presupuestaria) en el periodo 2017. Se utilizó la guía de análisis documental, donde se incluyó presupuesto programado y ejecutado de ingresos y gastos de la fuente de financiamiento de recursos directamente recaudados, presupuesto programado y ejecutado de ingresos y gastos fuente de financiamiento de recursos por operaciones oficiales de crédito, presupuesto programado y ejecutado de ingresos y gastos de fuente de financiamiento donaciones y transferencias, presupuesto programado y ejecutado de ingresos y gastos fuente de financiamiento fondo de compensación municipal, presupuesto programado y ejecutado de ingresos y gastos fuente de impuestos municipal así como también el uso de los fondos públicos de las diferentes fuentes.

\section{RESULTADOS}

En la tabla 01 se evidencia la evolución del Presupuesto de Apertura, el punto más alto fue el año 2014 con S/. 37783006 soles y el punto más bajo fue el año 2017 con S/. 18547510 soles. El Presupuesto Institucional Modificado, el punto más alto fue el año 2014 en S/. 60360199 soles y el punto más bajo fue el año 2017 con S/. 26413237 soles.

\begin{tabular}{lll}
$\begin{array}{l}\text { Tabla } 1 \\
\text { Programación presupuestaria (Ingresos) }\end{array}$ \\
\hline AÑO & PROG. PRESUP. (PIA) & \\
\hline 2014 & 37783006 & PROG. PRESUP. (PIM) \\
2015 & 25763736 & 60360199 \\
2016 & 25514125 & 32983727 \\
2017 & 18547510 & 36727873 \\
\end{tabular}

Fuente: MEF (2017)

Cabe indicar que al año 2014 el PIM fue superior al PIA registrando un incremento del 59,75\%, pero el nivel de inversión considerando a la ejecución presupuestaria respecto al PIM alcanzó el 91,5\%. Seguidamente en el año 2015 el PIM fue superior al PIA registrando un incremento del 28,02\%, pero el nivel de inversión considerando a la ejecución presupuestaria respecto al PIM alcanzó el 72,5\%. En el año 2016 el PIM fue superior al PIA registrando un incremento del 43,95\%, pero el nivel de inversión considerando a la ejecución presupuestaria respecto al PIM alcanzó el 87,2\% y en el año 2017 el PIM fue superior al PIA registrando un incremento del 42,40\%, pero el nivel de inversión considerando a la ejecución presupuestaria respecto al PIM alcanzó el 76,5\%.

En cuanto en la distribución de los gastos para el año 2014 se tiene S/. 927138 para las enfermedades meraxénicas y zoonosis, para la reducción de delitos y faltas que afecta la seguridad ciudadana se tiene $S /$. 316 655. En lo referente a la gestión integral de residuos sólidos S/. 86 966, en la mejora de la sanidad 
animal en S/. 666 323, en la mejora de mantenimiento de la sanidad vegetal en S/. 842 262, en el aprovechamiento de los recursos hídricos para uso agrario en S/. 196 419, en lo referente a la reducción del costo, tiempo e inseguridad vial en el Sistema de transporte no registra monto, en la reducción de vulnerabilidad y atención de emergencias por desastres en S/. 8749558 soles, para el programa nacional de saneamiento rural en S/. 8448 375, para los logros de aprendizaje de estudiantes de la educación básica regular en S/. 351 941, en el mejoramiento integral de barrios no presenta monto, la mejora de la competitividad de los destinos turísticos no presenta monto, en el incremento de la práctica de actividades físicas, deportivas y recreativas no presenta monto, en las acciones centrales en S/. 1765956 y las asignaciones presupuestarias que no resultan en productos en S/. 32901981 soles.

En cuanto en la distribución de los gastos para el año 2015 no se tiene monto para las enfermedades meraxénicas y zoonosis, para el programa articulado nutricional no hay presupuesto, para la reducción de delitos y faltas que afecta la seguridad ciudadana se tiene S/. 721 034, en lo referente a la gestión integral de residuos sólidos S/. 29 941, en la mejora de la sanidad animal en S/. 939 956, en la mejora de mantenimiento de la sanidad vegetal en S/. 52 482, en el aprovechamiento de los recursos hídricos para uso agrario en S/. 272 825, en lo referente a la reducción del costo, tiempo e inseguridad vial en el Sistema de transporte S/. 1343 285, en la reducción de vulnerabilidad y atención de emergencias por desastres en S/. 450 464, para el programa nacional de saneamiento rural en S/. 446625 soles, para los logros de aprendizaje de estudiantes de la educación básica regular en S/. 6405 soles, en el mejoramiento integral de barrios no presenta monto, la mejora de la competitividad de los destinos turísticos no presenta monto, en el incremento de la práctica de actividades físicas, deportivas y recreativas S/. 844 614, en las acciones centrales en S/. 1345755 y las asignaciones presupuestarias que no resultan en productos en S/. 17451711 soles.

En cuanto en la distribución de los gastos para el año 2016 no se tiene monto para las enfermedades meraxénicas y zoonosis, para el programa articulado nutricional en S/. 5 180, para la reducción de delitos y faltas que afecta la seguridad ciudadana se tiene S/. 1024 608, en lo referente a la gestión integral de residuos sólidos S/. 21 455, en la mejora de la sanidad animal en S/. 33 339, en la mejora de mantenimiento de la sanidad vegetal en S/. 2305 soles, en el aprovechamiento de los recursos hídricos para uso agrario en S/. 168082 soles, en lo referente a la reducción del costo, tiempo e inseguridad vial en el Sistema de transporte S/. 2669 377, en la reducción de vulnerabilidad y atención de emergencias por desastres en S/. 87 543, para el programa nacional de saneamiento rural sin monto, para los logros de aprendizaje de estudiantes de la educación básica regular en S/. 51 662, en el mejoramiento integral de barrios S/. 54 392, la mejora de la competitividad de los destinos turísticos 145 954, en el incremento de la práctica de actividades físicas, deportivas y recreativas S/. 1796 374, en las acciones centrales en S/. 1 455163 y las asignaciones presupuestarias que no resultan en productos en S/. 18129364.

En cuanto en la distribución de los gastos para el año 2017 no se tiene monto para las enfermedades meraxénicas y zoonosis, para el programa articulado nutricional en S/. 1 200, para la reducción de delitos y faltas que afecta la seguridad ciudadana se tiene S/. 818 369, en lo referente a la gestión integral de residuos sólidos S/. 21 455, en la mejora de la sanidad animal no tiene monto, en la mejora de mantenimiento de la sanidad vegetal no tiene monto, en el aprovechamiento de los recursos hídricos para uso agrario en S/. 151 588, en lo referente a la reducción del costo, tiempo e inseguridad vial en el Sistema de transporte sin monto, en la reducción de vulnerabilidad y atención de emergencias por desastres sin monto, para el programa nacional de saneamiento rural sin monto, para los logros de aprendizaje de estudiantes de la educación básica regular en S/. 2190 536, en el mejoramiento integral de barrios 769886 soles, la mejora de la competitividad de los destinos turísticos sin monto, en el incremento de la práctica de actividades físicas, deportivas y recreativas S/. 1565 629, en las acciones centrales en S/. 1516524 y las asignaciones presupuestarias que no resultan en productos en S/. 13139 676.

Respecto a la programación presupuestaria de ingresos por Fuente de financiamiento, periodo 20142017, en Recursos Directamente Recaudados, para el año 2014 se tiene S/ 5914764 soles, para el año 
2015 en S/. 3925 020, el año 2016 en S/. 43335677 y para el año 2017 en S/. 3817151 soles. En recursos por operaciones oficiales de crédito, para los años 2014, 2015 y 2016 no se tiene monto y para el año 2017 se tiene S/. 1112 305. En donaciones y transferencias, para el año 2014 se tiene S/. 67357 soles, el año 2015 se tiene S/. 73 407, para el año 2016 se tiene S/. 30000 y para el año 2017 se tiene la cantidad de S/. 57370 y en recursos determinados: Para el año 2014 se tiene S/. 54298975 soles, el año 2015 se tiene S/. 28923 018, el año 2016 se tiene S/. 32299914 y para el año 2017 se tiene S/. 21360 329 (tabla 02)

Tabla 2

Programación presupuestaria de ingresos por fuente de financiamiento, periodo 2014-2017 en soles

\begin{tabular}{lllll}
\hline Fuente de Financiamiento & 2014 & 2015 & 2016 & 2017 \\
\cline { 2 - 5 } & TOTAL S/. & TOTAL S/. & TOTAL S/. & TOTAL S/. \\
\hline $\begin{array}{l}\text { Recursos Directamente Recaudados } \\
\text { Recursos por operaciones oficiales } \\
\text { de crédito }\end{array}$ & 0 & 314764 & 4335677 & 317151 \\
Donaciones y transferencias & 67357 & 0 & 0 & 112305 \\
Recursos Determinados & 54298975 & 73407 & 30000 & 57370 \\
TOTAL & 60281096 & 32921445 & 36665591 & 21360329 \\
\hline
\end{tabular}

Fuente: MEF (2017)

La Municipalidad Distrital de Ite tuvo ingresos por grupo genérico presentadas en la tabla 03

Tabla 3

Ingresos por grupo genérico de la Municipalidad Distrital de Ite año 2017 en soles

\begin{tabular}{llll}
\hline Genérica & \multicolumn{1}{c}{ PIA } & \multicolumn{1}{c}{ PIM } & Recaudado \\
\hline Impuestos y contribuciones obligatoria & 41806 & 41806 & 17077 \\
Venta de bienes y servicios y derechos & 3810496 & 3810496 & 1766116 \\
administrativos & & & \\
Donaciones y transferencias & 21599541 & 22594147 & 22441904 \\
Otros ingresos & 0 & 30000 & 836368 \\
Venta de activos no financieros & 0 & 0 & 28310 \\
Saldos de balance & 0 & 10189142 & 11041776 \\
TOTAL & 25451843 & 36665591 & 36131551 \\
\hline
\end{tabular}

De los proyectos de inversión de la Municipalidad Distrital de Ite para el año 2017, se lograron ejecutar al 100\%; cinco (5) proyectos de inversión, ejecución por debajo del 100\%, veinte (20) proyectos de inversión y proyectos que no se lograron ejecutar, uno (1). Los proyectos que más sobresalen son: Mejoramiento de la prestación de servicios de apoyo a la comercialización de los productos agrícolas con potencial agroexportable; mejoramiento de la competitividad de la cadena productiva del ganado bovino, mejoramiento del servicio de maquinaria agrícola en la división de servicios agropecuarios y mejoramiento del servicio de los espacios públicos (parques y jardines). El presupuesto programado y ejecutado de ingresos y gastos por fuente de financiamiento en lo que se refiere al presupuesto de ingresos programado se tiene en PIM S/. 26347155 soles y lográndose una recaudación de S/. 27303756 soles, que representa el 103,6\% llegando a superar a lo programado; en cuanto a los gastos se programó en PIM S/. 26347155 soles, llegándose a ejecutar S/. 20139382 soles, que representa el 76,4\% del presupuesto programado. 
Para el presupuesto programado y ejecutado de ingresos y gastos influye significativamente en el uso de los fondos públicos, debido a que en este rubro se hizo una programación de ingresos de S/. 1112305 soles, lográndose una recaudación efectiva de S/. 2100000 soles: como se aprecia se logró captar más de lo programado que representa el 188.79\%; por lo que en esta fuente se programó un presupuesto de gasto de S/. 1112305 soles, y se ejecución solo S/. 553496 soles, lo que demuestra que aún no se logra cumplir con el total del presupuesto de gastos que representa un $49,8 \%$.

El presupuesto programado y ejecutado de ingresos y gastos de fuente de donaciones y transferencias en la Municipalidad Distrital de Ite, 2017, debido a que en este rubro se hizo una programación de ingresos de S/. 57370 soles, lográndose una recaudación efectiva de S/. 82926 soles, se aprecia se lograron captar más de lo programado que representa el $144,5 \%$.

En el uso de compensación municipal se hizo una programación de ingresos de S/. 434170 soles, lográndose una recaudación efectiva de S/. 420432 soles, y se ejecutó lo programado, con el 96,8\%. En esta fuente se programó un presupuesto de gasto de S/. 434170 soles, del cual se logró ejecutar S/. 391 925 soles, y se ejecutó casi con el total del presupuesto de gastos $(90,3 \%)$.

El presupuesto de impuesto municipal se hizo una programación de ingresos de S/. 41806 soles, lográndose una recaudación efectiva de S/. 108821 soles, como se aprecia se lograron captar más de lo programado que representa el 260.3\%; por lo que en esta fuente se programó un presupuesto de gasto de S/. 41806 soles, y solo se ejecutó S/. 9537 soles, lo que demuestra que aún no se logra cumplir con el total del presupuesto de gastos que representa un $22.8 \%$.

El presupuesto programado de fuente Canon y sobrecanon, regalías, renta de aduanas se hizo una programación de ingresos de S/. 20884353 soles, lográndose una recaudación efectiva de S/. 21674184 soles, como se aprecia se lograron captar más de lo programado que representa el $103.8 \%$. En esta fuente se programó un presupuesto de gasto de S/. 20884353 soles, y se ejecutó S/. 16890728 soles, lo que demuestra que aún no se logra cumplir con el total del presupuesto de gastos, que representa un $80.9 \%$.

\section{DISCUSIÓN}

Los hallazgos detectados se parecen a lo establecido por Gancino (2010), quien concluye que la planificación presupuestaria que se desarrolla sin el personal preparado, solo que se basan en la experiencia, no contribuye a que desarrollen sus actividades en forma eficaz, según la percepción de los colaboradores de los entes organizativos; quienes asumen que el $61.70 \%$ de los recursos asignados, son entregados a tiempo; sin embargo, no satisfacen las necesidades porque no se distribuyen de manera adecuada. Los resultados encontrados son equivalentes con lo informado por Matamoros (2013), quien concluye que la planificación contribuye a que el ente municipal tenga mejores resultados; especialmente en temas presupuestarios, planificación del mantenimiento y de la ejecución de los proyectos y en el cumplimiento de los objetivos y planes trazados, de tal forme que se tome las acciones correctivas en el momento adecuado y lo informado por Montejo y Arones (2014), quien concluyó que no existen diferencias significativas en la Genérica de Gasto 2.3 de Bienes y Servicios en cuanto a lo programado y lo modificado en el Ministerio Público 2011 al 2013. En los ejercicios fiscales en los periodos individualizados 2011 al 2013, no están relacionadas la programación y las modificaciones presupuestarias, debido a que dentro la programación se considera lo proyectado y evaluado por la Gerencia de Programación, teniendo un nivel de inconsistencia.

La Gerencia de Programación solicita modificaciones presupuestales en forma desmedida y sólo para cubrir necesidades momentáneas. Por tanto, no es pertinente que exista muchas modificaciones presupuestales, debido a que implica que cuando se haga la evaluación presupuestaria, sería más complicado desarrollar un buen control; tal aspecto sucede porque los directivos no priorizan el desarrollo 
de una buena programación presupuestal. En contraste a nuestros resultados, Lozano (2016), concluye que no constituye herramienta de articulación entre la prospectiva de programación presupuestaria vigente con el plan de desarrollo regional concertado que coadyuve en el proceso de desarrollo concertado de la región Ucayali, debido a que la eficacia; técnicas y procedimientos; análisis, valoración y recomendaciones, y los objetivos y metas no son concordantes con el desarrollo regional que la población demanda; y a la prueba de hipótesis que recomienda implementar una nueva herramienta de articulación. No es eficiente la articulación de la eficacia entre la prospectiva de programación presupuestaria vigente y el logro de los objetivos del plan de desarrollo regional concertado que incida en el proceso de desarrollo concertado de la región Ucayali, debido a que está en algo de acuerdo y representa un $36.23 \%$. Por tanto, la programación presupuestaria es un proceso crucial para el desarrollo de una buena ejecución presupuestal, por lo que es primordial que las unidades orgánicas desarrollan adecuados requerimientos, que se considere en el plan operativo en forma correcta, para que no haya modificaciones. Coaquira (2017), observa en su trabajo que la asignación presupuestaria influye en forma significativa en la gestión del Área de Transporte Público y Tránsito en la Municipalidad Provincial de Tacna. La asignación presupuestaria para recursos materiales influye en forma significativa en el nivel de eficiencia del Área de Transporte Público y Tránsito en la Municipalidad Provincial de Tacna. Por tanto, se determina que todas las unidades orgánicas requieren desarrollar en forma eficiente sus actividades, sin embargo, no pueden hacer porque no le asignan en forma correcta el presupuesto, debido a que no hay una correcta programación del presupuesto, pues a veces se asigna por criterios políticos.

Se recomienda al Alcalde Distrital de Ite disponga la elaboración de un Plan de fortalecimiento de Control interno al proceso de programación presupuestaria para optimizar el uso de los fondos públicos. El Jefe del Área de Presupuesto de la Municipalidad Distrital de Ite, debería implementar el Plan de fortalecimiento de Control Interno de la programación de la fuente de financiamiento: recursos directamente recaudados para mejorar el uso de los fondos públicos. Se recomienda al El Jefe del Área de Presupuesto de la Municipalidad Distrital de Ite, desarrolle un monitoreo permanente sobre la programación de la fuente de financiamiento: recursos por operaciones oficiales de crédito para optimizar el uso de fondos públicos. Se hace necesario que el Jefe del Área de Recursos Humanos de la Municipalidad Distrital de Ite, desarrolle un programa de capacitación sobre la programación de la fuente de financiamiento: donaciones y transferencias para optimizar el uso de los fondos públicos.

\section{REFERENCIAS BIBLIOGRÁFICAS}

Andia, W. (2011). Planeamiento estratégico del sector público. Lima: El saber.

Apaza, R. (2010). Evaluación de la Ejecución Presupuestaria y el Cumplimiento de Metas Presupuestarias de la Dirección Regional Agraria Puno, Periodos 2008 y 2009. (Tesis de grado). Universidad Nacional del Altiplano.

Ayala, F. y Pineda, H. (2016). Evaluación de la ejecución presupuestaria y el cumplimiento de Metas y Objetivos de la Municipalidad Distrital de Kelluyo, Periodos 2014 -2015. (Tesis de grado).

Carrasco, S. (2006). Metodología de la investigación científica. Editorial San Marcos. Lima

Coaquira, B. (2017). Asignación presupuestaria y su influencia en el nivel de gestión del área de transporte público y tránsito en la municipalidad Provincial de Tacna, año 2015. (Tesis de grado). Universidad José Carlos Mariátegui - Moquegua

De la Cruz, W. (2018). Programación presupuestaria y su relación con el nivel de inversión pública en el gobierno regional de La Libertad: 2011 - 2016. (Tesis de grado). Universidad César Vallejo.

Gancino, A. (2010). La planificación presupuestaria y su incidencia en la información financiera de la Fundación Pastaza trabajo de graduación. (Tesis de grado). Universidad Técnica de Ambato Ecuador.

Glosario de términos presupuestales (2017). Oficina de Planeamiento Estratégico. Recuperado de http://www.hsr.gob.pe/transparencia/pdf/2013/ejecucion_presupuesto_glosario_terminos.pdf 
Hernández, R.; Fernández, C.; Baptista, R. (2014). Metodología de la Investigación. México: Editorial. Mc. Graw Hill

Lozano, L. (2016). Propuesta prospectiva de programación presupuestaria para el proceso de desarrollo concertado de la región Ucayali. (Tesis de doctorado). Universidad Nacional Hermilio Valdizan.

Mamani (2016). Aplicación de las normas generales de tesorería y su influencia en el manejo de los fondos públicos de la Unidad de Gestión Educativa Local de Carabaya Períodos 2014 2015. (Tesis de grado). Universidad José Carlos Mariátegui - Moquegua.

Matamoros, Y. (2013). Propuesta para la implementación de un sistema de control interno presupuestario para la gestión presupuestaria de la Municipalidad Distrital de Pancan. (Tesis de grado). Universidad Nacional del Centro. (Huancayo).

Méndez, C. (2012). Metodología de la investigación. Colombia.

Montejo, M. y Arones, N. (2014). Estudio descriptivo comparativo de la programación y modificaciones presupuestarias de la genérica de gasto 2.3 bienes y servicios del Ministerio Público 2011 al 2013. (Tesis de maestría). Universidad César Vallejo.

Palacios (2012). El presupuesto participativo basado en resultados como instrumento para la toma de decisiones en la asignación y ejecución del presupuesto de inversiones de la municipalidad distrital de Casa Grande, 2012. (Tesis de grado). Universidad Privada Antenor Orrego - Trujillo.

Paredes, F. (2006). Presupuesto Público. Aspectos Teóricos y Prácticos. Universidad de Los Andes, Mérida, Venezuela

Ruly N. (2012). El Plan Operativo Institucional. Universidad Nacional de San Agustín Arequipa.

Sainz de Vicuña, J.M. (2015). El plan estratégico en la práctica. $4^{\circ}$ ed. España: Business marketing school.

Supo, H. A. (2015). Evaluación de la Ejecución Presupuestaria y el Cumplimiento de Metas del Hospital Regional Manuel Núñez Butron, periodos 2012-2013. (Tesis de grado). Universidad Nacional del Altiplano. Puno.

Villegas, J. (2016). Caracterización de las fuentes de financiamiento y su incidencia en la ejecución presupuestaria de las municipalidades del Perú. Caso: Municipalidad Provincial de Piura, año 2015. (Tesis de grado). Universidad Católica Los Ángeles de Chimbote.

NORMAS

Ley № 28411, Ley General del Sistema de Presupuesto Público.

Ley Anual de Presupuesto del Sector Público para el Año Fiscal.

Ley de Equilibrio Financiero.

Ley de Endeudamiento.

Ley de Responsabilidad y Transparencia Fiscal - Ley $N^{\circ} 27245$ modificada por Ley $N^{\circ} 27958$ y su Reglamento.

Ley Orgánica de Municipalidades - Ley № 27972.

Ley de Bases de la Descentralización - Ley N² 27783.

Ley de Tributación Municipal - D. Leg. N 776 y normas modificatorias.

Ley del Sistema Nacional de Inversión Pública - Ley N²7293 modificada por la sexta disposición complementaria de la ley No 28522 y la Ley N²8802, y su Reglamento.

Ley Marco del Presupuesto Participativo - Ley N²8056 y su Reglamento.

Normas que definen conceptos vinculados a las transferencias de fondos públicos a los gobiernos locales (FONCOMUN, Canon y Sobrecanon, Regalías, Participación en Renta de Aduanas, Programa del Vaso de Leche, etc.). 